
allemande

47-1| 2015

L'Allemagne et l'Europe | La déprise de l'Empire napoléonien en Allemagne en 1813

Réciter, chanter, mettre en scène. Les enjeux des " guerres de libération » dans la culture scolaire en Allemagne (1814-1913)

\title{
Bettina Severin-Barboutie
}

\section{OpenEdition}

\section{Journals}

Édition électronique

URL : https://journals.openedition.org/allemagne/479

DOI : 10.4000/allemagne.479

ISSN : 2605-7913

Éditeur

Société d'études allemandes

Édition imprimée

Date de publication : 26 juin 2015

Pagination : 195-208

ISSN : 0035-0974

Référence électronique

Bettina Severin-Barboutie, «Réciter, chanter, mettre en scène. Les enjeux des " guerres de libération » dans la culture scolaire en Allemagne (1814-1913) », Revue d'Allemagne et des pays de langue allemande [En ligne], 47-1 | 2015, mis en ligne le 13 décembre 2017, consulté le 19 mai 2021. URL : http:// journals.openedition.org/allemagne/479; DOI : https://doi.org/10.4000/allemagne.479 


\section{Réciter, chanter, mettre en scène. Les enjeux des " guerres de libération » dans la culture scolaire en Allemagne (1814-1913)}

\section{- Bettina Severin-Barboutie *}

Tout comme la famille, les écoles sont des lieux privilégiés pour l'enculturation des jeunes générations. C'est à l'école que celles-ci doivent s'approprier, du moins en partie, les normes, valeurs, règles, savoirs, codes et symboles propres à leur culture et apprendre à les appliquer. C'est à l'école également qu'elles sont initiées aux traditions et au passé de leur culture. C'est donc à l'école que les enfants sont à la fois intégrés à une communauté historique imaginée (telle que la nation) et conditionnés à s'identifier à elle, qu'ils s'approprient son langage et ses codes spécifiques et apprennent des règles de conduite. Ainsi, c'est à l'école que se prépare la société de demain. Valable pour les établissements scolaires d'aujourd'hui autant que pour ceux d'autrefois, cette double fonction place l'école au cœur de la construction du monde social ${ }^{(1)}$.

Malgré la place centrale de l'école dans la vie des enfants et la (sur)vie des sociétés, le rôle formateur et identitaire des établissements scolaires dans le passage des jeunes vers l'âge adulte ne constitue pas, jusqu'ici, un objet d'étude privilégié de la part des historiens. Il est vrai que les analyses des représentations historiques dans les manuels scolaires se sont multipliées ces dernières années tout comme celles des événements commémoratifs scolaires et l'influence politique de l'enseignement sur les enfants $^{(2)}$. Néanmoins, la question de l'enculturation à proprement parler est encore

* Boursière Feodor Lynen de la Fondation Alexander von Humboldt, Irice/LabEx Écrire une Histoire nouvelle de l'Europe, chercheur invitée à l'IHA PAris.

1 Cf. Anja Richter, Inszenierte Bildung. Festkultur im 19. Jahrhundert, Iéna, Edition Paideai, 2010, p. 16.

2 Pour les recherches historiques sur les manuels scolaires, voir notamment la bibliographie accessible sur le site de l'Institut Georg-Eckert à Braunschweig ainsi que les projets de recherche de ce dernier, URL: http://www.gei.de/nc/georg-eckert-institut.html (05.12.2013); quant aux fêtes scolaires, voir A. Richter, Bildung (note 1); Hartmut Heller, "Schulfeste und Schulbräuche ", in: Max Liedtke (dir.), Handbuch der Geschichte des Bayerischen Bildungswesens, t. 4, Bad Heilbrunn/Obb., C.H. Beck, 
très peu posée, du moins explicitement ${ }^{(3)}$. C'est également le cas de la question qui nous intéresse ici, à savoir celle des enjeux des "guerres de libération " de 1813 dans la culture scolaire en Allemagne au XIX ${ }^{\mathrm{e}}$ siècle. Quasiment absent des travaux historiques même les plus récents ${ }^{(4)}$, le thème est englobé, la plupart du temps, dans des problématiques plus larges, telles que l'analyse des concepts de "Nationalerziehung " ou l'éducation "patriotique " des écoliers ${ }^{(5)}$, les représentations de Napoléon dans les manuels scolaires ${ }^{(6)}$, l'histoire des fêtes publiques au IIX $^{\mathrm{e}}$ siècle $^{(7)}$ ou le culte militaire ${ }^{(8)}$. Bien qu'indispensables pour la connaissance du sujet, ces études n'en éclairent qu'un champ limité et ceci parfois même de façon allusive. De surcroît, une partie de ces travaux se concentre sur l'histoire de l'Empire allemand sans réellement tenir compte des décennies précédentes, ou bien sur certains États, avant tout la Prusse, mais aussi la Bavière et la Saxe pour la relative autonomie régionale qui était la leur.

En résulte, pour notre analyse, l'impossibilité de brosser un tableau exhaustif de la transmission des "guerres de libération » dans les écoles allemandes. En revanche, il est nécessaire de transformer la question en objet d'étude véritable en cherchant à comprendre à quels moments, dans quelle mesure et pour quelles raisons les « guerres de libération" sont devenues un objet de transmission dans les écoles. Ainsi, nous proposons de prendre en compte, autant que possible, tout le $\mathrm{XIX}^{\mathrm{e}}$ siècle, à savoir la période entre la fin des conflits militaires en 1814-1815 et le déclenchement de la Première Guerre mondiale, en analysant, dans un premier temps, les rendez-vous des élèves avec les " guerres de libération » en dehors des établissements scolaires et, dans un deuxième temps, la

1997, p. 244-272; pour l'influence politique, voir Heinz SтüвIG, Nationalerziehung. Pädagogische Antworten auf die "deutsche Frage » im 19. Jahrhundert, Schwalbach/Ts., Wochenschau, 2006; Andrea Meissner, Die Nationalisierung der Volksschule. Geschichtspolitik im Niederen Schulwesen Preußens und des deutschsprachigen Österreich 1866 bis 1933/38, Berlin, Duncker \& Humblot, 2009.

3 Un exemple est le livre de A. Richter, Bildung (note 1).

4 Cf. le "Forum 'Befreiungskriege' ", URL: http://www.sehepunkte.de/2014/05/forum/befreiungskriege-182/ (16.05.2014).

5 H. StüBig, Nationalerziehung (note 2); A. MeIssner, Nationalisierung (note 2); Siegfried WeICHLEIN, Nation und Region. Integrationsprozesse im Kaiserreich, Düsseldorf, Droste, 2004, p. 323-328; Abigail Green, Fatherlands. State-Building and Nationhood in Nineteenth-Century Germany, Cambridge, Cambridge University Press, 2001, p. 189-222; Bernd SchönemanN, « Nationale Identität als Aufgabe des Geschichtsunterrichts nach der Reichsgründung », Internationale Schulbuchforschung, 1 (1989), p. 107-128.

6 Armin Rosenberger, Das Zeitalter Napoleons in den bayerischen und preußischen Schulgeschichtslehrbüchern des 19. Jahrhunderts bis zum Ende des Ersten Weltkriegs, Hausarbeit zur Erlangung des Diplomgrades, Munich, Universität der Bundeswehr, 1992.

7 Ute SchneIder, Politische Festkultur im 19. Jahrhundert. Die Rheinprovinz von der französischen Zeit bis zum Ende des Ersten Weltkrieges (1806-1918), Essen, Klartext, 1995; Simone Mergen, Monarchiejubiläen im 19. Jahrhundert. Die Entdeckung des historischen Jubiläums für den monarchischen Kult in Sachsen und Bayern, Berlin, Leipziger Universitätsverlag, 2005; Monika WiENForT, " Kaisergeburtstagsfeiern am 27. Januar 1907. Bürgerliche Feste in den Städten des Deutschen Kaiserreichs », in: Manfred Hettling, Paul Nolte (dir.), Bürgerliche Feste. Symbolische Formen politischen Handelns im 19. Jahrhundert, Göttingen, Vandenhoeck \& Ruprecht, 1993, p. 157-191 ; Wolfram SiEmANN, « Krieg und Frieden in historischen Gedenkfeiern des Jahres 1913 », in: ibid., p. 298-320.

8 Jakob Vogel, Der Kult der 'Nation in Waffen' in Deutschland und Frankreich, 1871-1914, Göttingen, Vandenhoeck \& Ruprecht, 1997; Heinz Lemmermann, Kriegserziehung im Kaiserreich. Studien zur politischen Funktion von Schule und Schulmusik 1890-1918, 2 tomes, Lilienthal, Brême, Eres Edition, 1984. 
rencontre des écoliers avec ces dernières au sein même de l'école dans l'enseignement, d'une part, et dans le cadre des fêtes scolaires et des occasions de commémorations, d'autre part, avec un intérêt tout particulier pour les fêtes monarchiques. Par leurs fonctions, leur déroulement ainsi que leur schéma interprétatif, ces fêtes représentaient des événements bien spécifiques: elles étaient autant des événements suggestifs ou de «closed texts", pour reprendre un terme d'Umberto Eco ${ }^{(9)}$, que des « rituels ", à savoir un « enchaînement d'actions marqué par la standardisation des formes, la répétitivité, des mises en scène, la performance ainsi qu'une symbolique et destiné à structurer le monde social " ${ }^{(10)}$. Étant donné le manque de travaux de recherches sur le sujet, nos propos ne présentent pas des résultats définitifs mais esquissent plutôt des pistes d'enquêtes pour des recherches futures et formulent des premières hypothèses à cet égard. Reste à signaler qu'en raison de la place limitée, l'article s'abstient de comparaisons avec des espaces non-germanophones bien que des études récentes sur la Grande-Bretagne par exemple invitent à l'élargissement du regard transversal en révèlant des analogies intéressantes au niveau des outils de travail utilisés dans les écoles en Grande-Bretagne tout comme dans l'Empire britannique durant le $\mathrm{XIX}^{\mathrm{e}}$ siècle ${ }^{(11)}$.

\section{Rendez-vous des écoliers avec les "guerres de libération " en dehors des établissements scolaires}

Dans l'immédiat après-guerre, les "guerres de libération » ne furent certainement pas enseignées ou même évoquées dans les écoles. D’une part, l’histoire enseignée à travers différentes matières et contenus était avant tout l'histoire biblique et/ou antique. D’autre part, pour les contemporains, les « guerres de libération » ne faisaient pas encore partie de l'histoire à cause de leurs conséquences sur le présent. Afin de ne pas entraîner les jeunes, disait un ancien élève de lycée hessois a posteriori, « dans des questions non résolues " ${ }^{(12)}$, il ne fallait pas les évoquer en cours.

C'est donc en dehors des salles de classe, au niveau local, que les premières générations d'élèves, c'est-à-dire: ceux qui avaient vécu au moment des conflits militaires rencontrèrent ce passé encore vivace, soit dans le cadre social des familles en raison de la présence de soldats ou de vétérans de guerre, de la perte d'un ou de plusieurs proches, des « objets » ou récits de guerre, etc., soit dans l'espace public à travers d'occasions commémoratives $^{(13)}$. Entre autres, les élèves étaient présents lors des inaugurations de monuments locaux en mémoire de la guerre en prenant en charge l'encadrement

9 Umberto Eco, Im Labyrinth der Vernunft, Leipzig, Reclam, 1995, p. 198-199; Birte Förster, « Popular History, Gender and Nationalism. Female Narratives of a National Myth ", in: Barbara KorTe, Sylvia Paletschek (dir.), Popular History Now and Then, Bielefeld, transcript, 2012, p. 149-168, ici p. 152-153.

10 Cf. Barbara Stollberg-Rilinger, Rituale, Francfort/New York, Campus, 2013, p. 9.

11 Cf. Stefanie Lethiridge, « Wars and 'Little' Heroes. Historical Topics in Popular Poetry Anthologies from the Nineteenth Century to the Present ", in: Korte/PaletscheK, History (note 9), p. 123-148, citation p. 125.

12 Jakob Loeber, Glückliche Gymnasiastenzeit. Erinnerungen aus meiner Schulzeit, Kiel, K. Beuck, 1925, p. 9.

13 Ute Planert, Der Mythos vom Befreiungskrieg. Frankreichs Kriege und der deutsche Süden. AlltagWahrnehmung - Deutung 1792-1841, Paderborn, Ferdinand Schöningh, 2007, p. 622-626, 630-632. 
musical de l'événement ${ }^{(14)}$. On les comptait également parmi les participants des fêtes organisées lors du premier anniversaire de la bataille de Leipzig, en octobre 1814. Ils célébraient la messe avec les adultes, chantaient et défilaient avec eux ${ }^{(15)}$.

Toutes ces rencontres avec les événements militaires de 1813 dans l'immédiat aprèsguerre étaient fortement basées sur la communication orale et symbolique, avaient un caractère intergénérationnel et représentaient des moments de mémoires partagées entre adultes et enfants. Toutefois, il n'allait pas de soi que les jeunes comprennent toujours ce qui se passait lors des festivités de 1814, des directeurs et professeurs d'école étant parfois obligés d'expliquer à certains élèves le sens de la fête ${ }^{(16)}$. Ainsi, le caractère éducatif des festivités prenait tout son sens.

La participation à la commémoration publique de la bataille de Leipzig resta l'expérience d'un nombre limité d'élèves, étant donné que par la suite, surtout après Carlsbad, l'événement ne fut plus commémoré qu'à certains endroits ${ }^{(17)}$. En revanche, la commémoration des "guerres de libération " ne s'éteignit pas pour autant. Bien au contraire: elle se diversifia et se multiplia au travers d'associations de vétérans et de femmes, la commémoration des morts, la multiplication de romans historiques et d'autres récits ou encore l'inauguration de monuments historiques en mémoire desdites guerres ${ }^{(18)}$. Par conséquent, la commémoration ne garda pas seulement son caractère intergénérationnel, mais les élèves continuèrent aussi à être confrontés aux événements à l'extérieur de l'école. Ainsi, par exemple, assistèrent-ils à l'inauguration de monuments historiques en mémoire des guerres tout au long du siècle, soit comme spectateurs, soit comme acteurs comme ce fut le cas des écoliers qui participèrent à l'inauguration de la "Befreiungshalle " à Kelheim par le roi de Bavière en $1863^{(19)}$. Étant donné que jusque dans la deuxième moitié du XIX ${ }^{\mathrm{e}}$ siècle, des vétérans de guerre, qu'Ernst Moritz Arndt nommait « les vieux » en $1863^{(20)}$, étaient considérés comme des " témoins vivants " pendant les commémorations publiques, ces événements représentèrent, pour plusieurs générations scolaires, des rencontres privilégiées avec la mémoire active et communicative ${ }^{(21)}$ des «guerres de libération ». De surcroît, tout comme les premières générations d'élèves, ces enfants pouvaient encore rencontrer les «guerres de libération » de façon plus ou moins implicite dans leur entourage, à travers un membre de la famille (père, oncle, etc.) ayant fait partie des volontaires en 1813 par

14 Karen Hagemann, "Männlicher Muth und teutsche Ehre ». Nation, Krieg und Geschlecht in der Zeit der antinapoleonischen Kriege Preußens, Paderborn, Ferdinand Schöningh, 2002, p. 501.

15 U. SCHNEIDER, Festkultur (note 7), p. 54.

16 U. Planert, Mythos (note 13), p. 619.

17 À Hambourg et Brême par exemple. Cf. Katherine B. AAslestad, « Remembering and Forgetting: The Local and the Nation in Hamburg's Commemorations of the Wars of Liberation ", Central European History, 38/3 (2005), p. 384-416, ici p. 394-395; Friedrich LenGer, « Die Erinnerung an die Völkerschlacht bei Leipzig im Jubiläumsjahr 1863 », in: Manfred Hett ling et al. (dir.), Figuren und Strukturen. Historische Essays für Hartmut Zwahr, Munich, KG. Saur, 2002, p. 25-41, ici p. 27 sq.

Ibid., p. 26-30; U. Planert, Mythos (note 13), p. 622-626, 630-632.

19 S. Mergen, Monarchiejubiläen (note 7), p. 173-174.

20 Anika Bethan, Napoleons Königreich Westphalen. Lokale, deutsche und europäische Erinnerungen, Paderborn, Ferdinand Schöningh, 2012, p. 78.

21 Cf. Jan Assmann, Das kulturelle Gedächtnis. Schrift, Erinnerung und politische Identität in frühen Hochkulturen, Munich, C.H. Beck, 1992, p. 50-51. 
exemple. Considérés comme une génération à part entière, ces «1813er ", comme on les appelait, gardaient, pendant de nombreuses années, un statut social privilégié dans la société locale, comme le montre l'illustre exemple de Theodor Fontane ${ }^{(22)}$. Enfin, l'entrée des "guerres de libération » dans les écoles à partir des années 1820 compléta l'intégration des enfants dans les commémorations publiques ou privées en dehors des écoles. Cette entrée se fit à trois niveaux: d'abord dans les fêtes scolaires et ensuite dans l'enseignement ainsi que dans les outils pédagogiques.

\section{Les enjeux des "guerres de libération » dans les écoles}

\section{Fêtes scolaires}

Dans les écoles de la Confédération germanique, c'est seulement au sein des établissements scolaires prussiens que la commémoration de la bataille de Leipzig devint un rituel dans le calendrier commémoratif, du moins pendant un demi-siècle. En effet, dès le début des années 1820, le gouvernement prussien ordonna que le 18 octobre soit célébré annuellement dans toutes les écoles de la monarchie sous forme de discours, récitations et lectures afin de réveiller l'amour et l'attachement des élèves pour la patrie prussienne. Cette décision transforma le caractère de la commémoration de façon profonde et durable. D’une fête publique et intergénérationnelle elle évolua vers une fête exclusivement réservée aux jeunes générations, et ceci d'autant plus que les fêtes scolaires organisées par la suite ne dépassaient guère le cadre scolaire. De surcroît, d'une fête nationale du peuple allemand elle évolua vers une fête de la monarchie prussienne. Cette tendance était d'autant plus marquée qu'elle fut accompagnée par d'autres fêtes liées à la monarchie prussienne, telles que la fête de l'anniversaire du roi de Prusse, célébrée également tous les ans depuis les années $1820^{(23)}$. Enfin, d'une fête de la «mémoire communicative » elle évolua vers une fête de la " mémoire culturelle » ${ }^{(24)}$.

Dans la deuxième moitié du $\mathrm{XIX}^{\mathrm{e}}$ siècle, la fête de la bataille de Leipzig et la commémoration des "guerres de libération " dans les écoles prussiennes évoluèrent au gré des changements politiques. L'acculturation patriotique des élèves se faisait donc dans un cadre de référence en mutation. Après la révolution de 1848, le gouvernement de Berlin décida de mettre les occasions commémoratives davantage au service de la patrie et de la transmission de l'Histoire avec un grand « $\mathrm{H}$ ». Par conséquent, les occasions de commémorer les "guerres de libération » se multiplièrent et le culte des " héros » et des " grands personnages » s'intensifia grâce à l'augmentation du nombre de personnes à qui on rendait hommage. C'est dans ce contexte que la reine Luise, bien qu'elle fût décédée avant les événements, fut introduite dans le cercle illustre des grands personnages à célébrer à l'école, notamment dans les écoles de filles ${ }^{(25)}$. Désormais, les

22 Theodor Fontane, Meine Kinderjahre, Fischer Taschenbuch Verlag, 2011, p. 107-110, ici p. 108; voir également Christopher CLARK, "The Wars of Liberation in Prussian Memory: Reflections on the Memorialization of War in Early Nineteenth-Century Germany », The Journal of Modern History, 68 (1996), p. 550-576, ici p. 556.

23 U. SCHNEIDER, Festkultur (note 7), p. 44-76, 80-98.

24 J. Assmann, Gedächtnis (note 21), p. 50-52.

25 Birte Förster, Der Königin Luise-Mythos. Mediengeschichte des "Idealbilds deutscher Weiblichkeit ", 1860-1960, Göttingen, V\&R unipress, 2011, p. 76-77, 124-132, 211-217, 230-233; H. LEMMERMANN, Kriegserziehung, t. 1 (note 8), p. 181-182; Karen Hagemann, « Heldenmütter, Kriegerbräute und 
« grands patriotes » n'étaient plus exclusivement masculins, guerriers ou monarques, mais comptaient aussi une femme dans leurs rangs dont la maternité et la charité complétaient les vertus des hommes et proposaient un modèle d'identification aux filles. Outre la multiplication et l'élargissement de la commémoration, le gouvernement prussien renforça son contrôle sur les écoles puisqu'il s'était avéré que les fêtes scolaires n’avaient pas toujours été célébrées comme Berlin l'aurait souhaité, que ce soit à cause de vacances, de la récolte, du manque d'intégration dans des festivités publiques ou des résistances ${ }^{(26)}$. Par conséquent, les écoles ne devaient plus se soustraire à leur obligation de célébrer les journées de commémoration imposées par le gouvernement.

Dans les anciens États membres de la Confédération du Rhin, la commémoration fut plus compliquée, même si l'anniversaire de la bataille continua à être célébré par certains. Cela concernait les anciens alliés de Napoléon dans leur ensemble et tout particulièrement ceux qui étaient restés fidèles à l'empereur français, jusqu'à sa défaite. Plusieurs modes opératoires ont été repérés par les historiens. La Saxe contournait les événements de 1813 catégoriquement, en construisant l'histoire du royaume à partir du retour du souverain en 1815. Ainsi, les élèves ne célébraient pas la bataille de Leipzig mais le retour de Frédéric-Auguste en juin 1815. Ils ne participèrent pas aux festivités autour du $50^{\mathrm{e}}$ anniversaire de la bataille de Leipzig en 1863 non plus, même si les directeurs et enseignants suivirent les événements de très près, un professeur de religion étant même membre du comité d'organisation du 19 octobre et honoré par le roi pour son engagement. De même, les élèves furent également absents lors des célébrations officielles du $50^{\mathrm{e}}$ anniversaire de la mort de Theodor Körner célébré la même année. S'étant porté volontaire pour la Prusse en 1813, cinquante ans plus tard, Körner était toujours associé à la défaite de la Saxe et ne pouvait donc pas servir de modèle aux élèves ${ }^{(27)}$. Dans le Mecklembourg, en 1863, les autorités interdisaient également aux lycéens de participer à la commémoration de la mort de Körner, mais pour d'autres raisons. Elles anticipaient des discours « démocratiques » et craignaient que ces derniers pussent avoir un effet néfaste sur les élèves ${ }^{(28)}$. En Bavière, la compromettante alliance avec la France et les luttes de 1813 furent intégrées dans une histoire dynastique longue. Cela permettait de les passer sous silence au moment des commémorations officielles et des fêtes scolaires organisées lors des anniversaires monarchiques, sans les ignorer totalement ${ }^{(29)}$. À Hambourg, l'appropriation des « guerres de libération » était encore différente. Étant considérés comme un nouveau point de départ pour la ville, les événements de 1813 y donnèrent lieu à la naissance d'un autre «mythe de la libération »: ils signifiaient la « reconquête de l'indépendance » par les citoyens

Amazonen. Entwürfe 'patriotischer' Weiblichkeit zur Zeit der Freiheitskriege ", in: Ute FrEvERT (dir.), Militär und Gesellschaft im 19. und 20. Jahrhundert, Stuttgart, Klett-Cotta, 1997, p. 174-200, ici p. 187-189.

U. SCHNEIDER, Festkultur (note 7), p. 93.

27 Pour la biographie de Theodor Körner, voir René Schilling, "Kriegshelden ». Deutungsmuster heroischer Männlichkeit in Deutschland 1813-1945, Paderborn, Ferdinand Schöningh, 2002, p. 33-36.

28 Ibid., p. 149.

29 Katherine B. Aaslestad, « Krieg, Demobilisierung und Erinnerungskultur in den republikanischen Stadtstaaten Hamburg, Bremen und Lübeck », in: Jörg Echternkamp, Kriegsenden, Nachkriegsordnungen, Folgekonflikte. Wege aus dem Krieg im 19. Jahrhundert, Fribourg-en-Brisgau, Rombach, 2011, p. 53-74, ici p. 71. 
de la ville eux-mêmes, donc ils étaient le fruit d'un véritable combat pour la liberté( ${ }^{(30)}$. Dans un premier temps, cette liberté fut célébrée à deux dates différentes: le 18 octobre et le 18 mars, le jour de la libération de la ville. C'est seulement en 1863 que le Sénat remplaça la célébration du 18 octobre par celle du 18 mars $^{(31)}$. Les écoles ne furent pas seulement intégrées dans les festivités officielles de cette journée commémorative, mais les professeurs contribuèrent également à répandre le mythe de la "libération » de la ville au sein de leur établissement et l'exemple d'un professeur du prestigieux lycée Johanneum révèle l'ancrage idéologique de certains enseignants. Reprenant la fameuse phrase "Le roi appela et ils accoururent tous " dans son discours devant les élèves, il proclama "Nous criâmes "Nous voulons être libres", et nous devînmes libres " ${ }^{(32)}$. Notons que de semblables efforts de reconstruction se rencontrent chez d'autres membres de la Confédération du Rhin, ainsi dans le Wurtemberg ${ }^{(33)}$.

Sous le Reich bismarckien et wilhelmien, la commémoration des « guerres de libération » semblait disparaître de la vie scolaire au profit de la commémoration de la guerre de 1870/71, plus apte, paraîtra-t-il, à « imprégner» les jeunes esprits. Mais cette disparition s'avéra trompeuse. D’une part, la commémoration revint de façon indirecte dans les écoles dans le cadre de la fête de l'anniversaire de l'empereur le 27 janvier ainsi que de la fête de la bataille victorieuse de Sedan le 2 septembre ${ }^{(34)}$. S'établissant successivement dans le calendrier commémoratif annuel des établissements scolaires de quasiment tous les États sauf de la Bavière ${ }^{(35)}$, ces deux fêtes devinrent même des lieux de mémoire privilégiés de l'ensemble des écoles en donnant l'occasion de rappeler les événements de 1813 à travers discours, chants et poèmes ${ }^{(36)}$. D'autre part, en 1913, la bataille de Leipzig fut de nouveau célébrée en grande pompe dans l'ensemble du Reich, depuis les institutions scolaires jusqu'à l'espace public. Y participèrent également, pour la première fois, des élèves de la Saxe ${ }^{(37)}$.

En revanche, depuis 1870 , le cadre dans lequel se déroulaient toutes ces fêtes avait profondément changé. Tout d'abord, l'issue de la guerre de 1870-1871 représentait une victoire pour tous les États allemands à l'exception de l'Autriche et ouvrait enfin la voie à l'unification rêvée depuis le début du siècle. Ensuite, l'arrivée de Guillaume II sur le trône intensifia l'emprise de l'État sur les écoles ${ }^{(38)}$. Enfin, les divisions politiques,

30 K.B. Aaslestad, « Remembering» (note 17), p. 400, 402, citation p. 400, note 64.

31 K.B. Aaslestad, « Krieg» (note 29), p. 71.

32 K.B. Aaslestad, « Remembering» (note 17), p. 400-403, citation p. 402.

33 U. Planert, Mythos (note 13), p. 634-641.

34 A. Richter, Bildung (note 1), p. 369.

35 H. Lemmermann, Kriegserziehung, t. 1 (note 8), note 35, p. 466.

36 Voir, à titre d'exemple, le programme des écoles supérieures de Goslar pour l'année scolaire 1890/1891. URL: http://digital.ub.uni-duesseldorf.de/ulbdsp/periodical/pageview/4637319?query=Schulfeier \%20befreiungskriege (31.10.2013); ainsi que A. Richter, Bildung (note 1), p. 369; M. WiENFORT, «Kaisergeburtstagsfeiern » (note 7), p. 177-180; Andrea Meissner, «Von der Waffenbrüderschaft zum Volkstum. Die Transformation des Nationalismus in bayerischen Volksschul-Lehrbüchern zwischen liberaler und katholischer Dominanz 1858-1933 ", Zeitschrift für bayerische Landesgeschichte, 70 (2007), p. 853-885, ici p. 862.

37 A. Richter, Bildung (note 1), p. 447-454.

38 Ibid., p. 376-381. 
économiques, sociales et culturelles étaient de plus en plus visibles ${ }^{(39)}$. S’y rajouta un changement moins apparent. Depuis la disparition des derniers vétérans des " guerres de libération » dans les années $1880^{(40)}$, l'anniversaire de la bataille de Leipzig ne dérivait plus que de la seule mémoire culturelle.

Le changement de contexte était en corrélation avec d'importantes mutations dans la représentation des "guerres de libération ». D’un événement prêtant à des interprétations variables ou concurrentes dans les différents États de la Confédération germanique, la bataille devint un succès incluant toutes les dynasties, marquant une étape historique primordiale dans le chemin du peuple allemand vers l'unité, ainsi qu'un point de cristallisation de l'image que ce dernier se faisait de lui-même (« la nation en armes ») et de l'autre (en l'occurrence de l'« ennemi » français) ${ }^{(41)}$. Ainsi, la bataille fut présentée aux jeunes générations comme un événement à la fois fédérateur, fondateur et identificateur de la "nation ", autrement dit un lieu de mémoire et d'émotion partagées ainsi qu'un futur commun en projet ${ }^{(42)}$. D’une défaite oubliée par certains, elle devint une victoire glorieuse et donc une ressource pour tous. Se généralisant à travers l'Allemagne impériale, c'est auprès des anciens vaincus comme la Saxe que ce schéma interprétatif en provenance de la Prusse produira la plus grande réévaluation. D’anciens vaincus, ces États devinrent des vainqueurs affirmés. Cependant, le processus d'hégémonie dont témoignèrent les célébrations scolaires n’aboutissait pas pour autant à un récit uniforme. Les différentes sous-narrations s'inséraient dans le récit glorieux de la résurrection de la Prusse, certes, mais n’y disparaissaient pas. À Hambourg par exemple, le rôle prépondérant de la ville dans sa propre libération fut maintenu ${ }^{(43)}$. Par conséquent, le récit d'une victoire commune chapeautait et intégrait mais ne remplaçait ni n'effaçait les différents récits en circulation, engendrant ce que Günther Lottes a appelé Leiterinnerung, il y a quelques années ${ }^{(44)}$.

Avec les représentations des "guerres de libération » les fonctions politiques des fêtes évoluèrent, elles aussi. Désormais les fêtes ne servaient plus seulement à l'intégration de tous les élèves ni à reconsolider, voire rétablir un ordre social de plus en plus troublé ${ }^{(45)}$. Mais elles devaient également prédisposer ou préparer filles et garçons au rôle spécifique qu'ils devaient occuper dans la société de demain. Ainsi, elles devaient unifier et diviser le monde social en même temps. La fête du centième anniversaire de

39 Friedrich Lenger, Dieter Langewiesche, "Urbanisierung und Binnenwanderung in Deutschland », in: F. Lenger (dir.), Stadt Geschichten. Deutschland, Europa und die USA seit 1800, Francfort-sur-leMain, Peter Lang, 2009, p. 30-43.

40 U. Planert, Mythos (note 13), p. 632.

41 J. Vogel, Kult (note 8), p. 170-178.

42 Kirstin Anne SchäfER, «Die Völkerschlacht», in: Étienne Françors, Hagen Schulze (dir.), Deutsche Erinnerungsorte II, Munich, C.H. Beck, 2003, p. 187-201; K. B. AASLESTAD, « Remembering» (note 17), p. 407.

43 Ibid., p. 407-416.

44 Voir Günter Lottes, «Erinnerung zwischen Psychologie und Kulturwissenschaft », in: Günter OesTERLE (dir.), Erinnerung, Gedächtnis, Wissen. Studien zur kulturwissenschaftlichen Gedächtnisforschung, Göttingen, Vandenhoeck \& Ruprecht, 2005, p. 163-184, ici p. 179.

45 Cf. Winfried Halder, Innenpolitik im Kaiserreich 1871-1914, Darmstadt, Wissenschaftlicher Buchverlag, 2011, p. 37-78 ; F. Lenger, "Erinnerung" (note 17), p. 25; Hans-Ulrich Thamer, Die Völkerschlacht bei Leipzig. Europas Kampf gegen Napoleon, Munich, C.H. Beck, 2013, p. 107-108. 
Theodor Körner dans les écoles prussiennes en 1891 fait preuve de cette ambivalence apparente ${ }^{(46)}$. En dressant aux élèves le tableau d'un héros-victime de guerre fort, loyal et mort pour sa patrie et en donnant à sa mort une signification transcendante ${ }^{(47)}$, la fête présentait la figure de Körner comme un modèle à imiter par les élèves masculins, un modèle d'homme d'ailleurs que nous retrouverons dans la commémoration des morts à la fin de la Grande Guerre ${ }^{(48)}$. En même temps, elle s'opposait aux revendications émancipatrices des femmes ${ }^{(49)}$.

Cependant, malgré la récupération systématique de l'événement par la culture scolaire sous l'Empire allemand, la commémoration festive des " guerres de libération » ne s'imposa pas à l'ensemble des écoles. D’abord parce que l'anniversaire de l'empereur et la bataille de Sedan ne furent pas fêtés partout, et ensuite parce qu'apparemment, certaines fêtes ne furent célébrées que dans un État et ne s'établirent pas ailleurs. À titre d'exemple, l'anniversaire de la reine Luise le 10 mars, la création de la Croix de fer (Eisernes Kreuz) par Frédéric-Guillaume III le même jour et la proclamation adressée à ses sujets (An mein Volk) le 17 mars furent exclusivement commémorés en Prusse ${ }^{(50)}$. Le centenaire de la naissance de Theodor Körner, le 26 août 1891, ne semble avoir été célébré que dans des écoles saxonnes et prussiennes avec une importante réévaluation du personnage, là encore surtout en Saxe ${ }^{(51)}$. Parallèlement, les fêtes monarchiques des différents Länder continuaient d'être honorées ${ }^{(52)}$. Continuèrent donc à coexister ou même à se concurrencer des commémorations de la patrie "intérieure » et extérieure au-delà de la création du Deuxième Reich ${ }^{(53)}$.

Par ailleurs, alors que nous savons ce que l'école était censée véhiculer, nous sommes dans l'incertitude, du moins à l'heure actuelle, au sujet de ce qu'elle réussissait à transmettre réellement, même si certains travaux historiques suggèrent qu'elle aurait eu une grande influence sur l'inconscient ainsi que sur l'orientation des élèves ${ }^{(54)}$. En premier lieu, il n'est pas possible de savoir combien d'élèves ont véritablement assisté aux fêtes. D'une part, la mise en place des fêtes monarchiques ne se fit que successivement dans les différents États de la Confédération germanique, parfois seulement dans la deuxième moitié du XIX ${ }^{\mathrm{e}}$ siècle. D’autre part, les fêtes imposées par les États furent parfois ignorées

46 A. Richter, Bildung (note 1), p. 438-440; René Schilling, « Die soziale Konstruktion heroischer Männlichkeit im 19. Jahrhundert. Das Beispiel Theodor Körner », in: Karen Hagemann, Ralf Pröve (dir.), Landsknechte, Soldatenfrauen und Nationalkrieger. Militär, Krieg und Geschlechterordnung im historischen Wandel, Francfort/New York, Campus, 1998, p. 121-144, ici p. 129-137.

47 Pour le culte de la mort ainsi que du sacrifice, voir B. Stollberg-Rilinger, Rituale (note 10), p. 78-84; U. Planert, Mythos (note 13), p. 627.

48 A. Richter, Bildung (note 1), p. 440; R. Schilling, « Kriegshelden» (note 27), p. 40-42.

49 R. SCHILling, "Konstruktion » (note 46), p. 135-137.

50 Bericht über das Schuljahr 1912 des Königlichen Gymnasiums Essen, URL: http://digital.ub.uniduesseldorf.de/ulbdsp/periodical/pageview/4302789?query=schulfeiern\%20befreiungskriege (22.11.2013); J. Vogel, Kult (note 8), p. 176-177; B. Förster, Königin (note 25), p. 131-132.

51 A. Richter, Bildung (note 1), p. 438-440; R. Schilling, « Kriegshelden » (note 46), p. 237.

52 H. Lemmermann, Kriegserziehung, t. 1 (note 8), p. 185.

53 Voir également les fêtes monarchiques dans les États-membres de l'Empire. A. Richter, Bildung (note 1), p. 356.

54 Hans-Ulrich Wehler, Das Deutsche Kaiserreich 1871-1918, Göttingen, Vandenhoeck \& Ruprecht, 1988 (6 éd.), p. 126; H. Lemmermann, Kriegserziehung, t. 1 (note 8), p. 190. 
ou provoquèrent des réticences, voire des résistances auprès de certains (sociaux-démocrates, professeurs, familles, etc.) tout au long du siècle, même là où l'État renforçait son contrôle durant le $\mathrm{XIX}^{\mathrm{e}}$ siècle. Ainsi, la réglementation et le contrôle officiels ne signifiaient pas nécessairement que les fêtes eurent réellement lieu, et lorsque ce fut le cas, les fêtes n'étaient pas toujours fréquentées par l'ensemble de la population scolaire ou bien elles ne perdurèrent pas, comme la journée de Sedan qui devint même insignifiante après $1900^{(55)}$. Elles pouvaient donc avoir un effet contreproductif: au lieu de renforcer la cohésion sociale et l'autorité monarchique au sein des écoles, elles pouvaient rompre l'unité des populations scolaires et même créer de nouvelles divisions.

Il apparaît ensuite que la résonance des fêtes auprès des élèves y ayant assisté est difficile à cerner. Certes, les écoliers étaient impliqués dans la chorégraphie des festivités et en furent même les principaux acteurs, que ce soit par des discours, la récitation de poèmes, le chant ou la mise en scène. Il est vrai aussi que certaines écoles s'approprièrent même le " mythe de la libération ", du moins officiellement. Ainsi, lors des célébrations du $100^{\mathrm{e}}$ anniversaire de la bataille en 1913, le lycée berlinois Zum Grauen Kloster incorpora sa propre histoire dans le schéma narratif de la libération, en faisant référence au grand nombre d'élèves du lycée qui se seraient portés volontaires en 1813 et en lisant un poème d'un de ces volontaires ${ }^{(56)}$. Toutefois, il est difficile de savoir s'il s'agissait d'un accomplissement d'exigences anticipées ou de réelles convictions, autrement dit: si nous sommes confrontés à une imprégnation effective ou prétendue, d'autant plus que des commentaires d'anciens élèves témoignent de la capacité de certains à « déchiffrer » les rituels imposés ${ }^{(57)}$. Reste à savoir dans quelle mesure ces fêtes pouvaient rallier des élèves immigrés d'autres régions de l'Empire, de plus en plus nombreux à certains endroits ${ }^{(58)}$.

Dans un troisième temps, c'est le cours de l'histoire lui-même qui semble nous inviter à la prudence. D’une part, la mise en service de ces célébrations scolaires ne trouva pas d'unanimité dans la société. Dans la ville de Brême par exemple, dans les années 1890, les sociaux-démocrates s'opposèrent ouvertement au caractère guerrier et anti-français de ces festivités ${ }^{(59)}$. D’autre part, les fêtes de plus en plus nombreuses ne permirent pas d'endoctriner la population de manière efficace à long terme. Sinon il n'y aurait pas eu la politisation des ouvriers, le conflit confessionnel, les revendications émancipatrices des femmes et des jeunes, etc.

Cependant, ce qui est sûr, c'est que, dans le courant du siècle, les conflits militaires de 1813 s'installèrent successivement mais durablement dans l'organisation de l'ensemble des fêtes scolaires à travers des chants et poèmes de Theodor Körner, d'Ernst Moritz Arndt et de Max von Schenkendorf ${ }^{(60)}$, mais aussi à travers la décoration des salles de

55 U. Schneider, Festkultur (note 7), p. 74-75, 93, 97-98, 144-148, 165-166; pour le déclin de la journée de Sedan, voir A. Richter, Bildung (note 1), p. 391-394.

56 Ludwig Martens, "Jahresbericht des Berlinischen Gymnasiums zum grauen Kloster (1914) ", in: H. Lemmermann, Kriegserziehung, t. 2 (note 8), p. 743-744, ici p. 743.

57 Ibid., doc. 84, p. 763 ; B. Förster, Königin (note 25), p. 217.

58 K.B. Aaslestad, «Remembering " (note 17), p. 406.

59 Cf. H. Lemmermann, Kriegserziehung, t. 2 (note 8), doc. 85, p. 764-769.

60 Ibid., doc. 53-57, p. 717-719, 721; voir également M. WienforT, « Kaisergeburtstagsfeiern » (note 7), p. 177-180. 
fêtes avec des feuilles de chêne, par exemple ${ }^{(61)}$. Dissociés de leur origine historique, la symbolique, la musique ainsi que les paroles et le langage des "guerres de libération » furent ainsi intégrés dans un répertoire de fête nationale standardisé et pérennisé, dans lequel les écoles de tous les États pouvaient puiser à n'importe quel moment du calendrier scolaire et pour n'importe quelle occasion sans que les élèves aient forcément connaissance des références historiques. Ainsi, elles devinrent un élément constitutif des rituels dans les écoles allemandes jusqu'au $\mathrm{xx}^{\mathrm{e}}$ siècle, sans que les populations scolaires aient nécessairement eu conscience de l'historicité des symboles. C'est peut-être dans cette transmission que se situe le véritable et durable « succès » de ces fêtes.

\section{Enseignement et outils pédagogiques}

Selon Heinrich Treitschke, une grande partie des élèves d'Allemagne aurait fait connaissance avec les grands héros prussiens des " guerres de libération » au fil de leur scolarité, et à l'en croire, cette « rencontre » aurait été efficace: "Ce n'est pas en vain, écrivit-il en 1870, que durant la longue période de paix des milliers de professeurs ont parlé de Stein et Blücher, ont évoqué des figures héroïques de la plus belle de nos guerres » ${ }^{(62)}$.

Si c'était à l'enseignement des "guerres de libération " en cours d'histoire à proprement parler que Treitschke faisait référence, ses propos étaient sans doute loin de la réalité. D’une part, l'enseignement de l'histoire ne s'installa véritablement quaprès 1850. Par conséquent, les "guerres de libération » n’ont pu vraiment entrer avant cette date dans le programme des écoles. D’autre part, cette entrée ne se fit pas de manière systématique, ni dans les établissements d'enseignement supérieur, et encore moins dans les écoles élémentaires où l'enseignement de l'histoire dépendait en grande partie de la volonté ainsi que de la qualité des professeurs desdites écoles. Ainsi, il faut supposer de grandes disparités d'un État à l'autre et même au sein d'un même État, tout au long du XIX ${ }^{\mathrm{e}}$ siècle. L'enseignement des " guerres de libération » dans une école ne reflétait absolument pas la situation générale. En Hesse électorale par exemple, le sujet était au programme dans un lycée de Hanau dans les années 1860 tandis quailleurs on ne l'évoquait même pas ${ }^{(63)}$.

En revanche, l'insertion des « guerres de libération » dans les outils pédagogiques se fit beaucoup plus tôt. En effet, dès les années 1820, elles firent leur apparition dans ces derniers, et ceci aussi bien dans les manuels d'histoire des anciens alliés de Napoléon qu'en Prusse et en Autriche. On les trouva alors dans les manuels pour les écoles supérieures des garçons et des filles ${ }^{(64)}$. Elles firent également partie des livres de lecture des écoles élémentaires dits Lesebücher. Quant à leur influence sur les élèves, elle est aussi difficile à évaluer que celle des fêtes.

61 U. Schneider, Festkultur (note 7), p. 94-98.

62 Heinrich von Treitschke, "Die Feuerprobe des norddeutschen Bundes ", Preußische Jahrbücher, 26/2 (1870), p. 242, cité d'après Michael JeISMANN, La patrie de l'ennemi. La notion d'ennemi national et la représentation de la nation en Allemagne et en France de 1792 à 1918, Paris, Éditions du CNRS, 1997 , p. 220.

63 J. Loeber, Gymnasiastenzeit (note 12), p. 9.

64 Voir le répertoire de l'ensemble des manuels scolaires d'histoire de Wolfgang JAcobmeyer, Das deutsche Schulgeschichtsbuch 1700-1945. Die erste Epoche seiner Gattungsgeschichte im Spiegel der Vorworte, t. 1, Berlin, LIT, 2011. 
Variant en fonction du type d'école, du sexe et de la confession visés, la production des livres scolaires dépendait en large partie de chaque État, de sa situation politique et dynastique ainsi que de son histoire proche et lointaine. Le récit, lui aussi, fut lié au territoire dans lequel ces livres devaient circuler. Ainsi, Der Kurhessische Kinderfreund, un manuel utilisé dans les écoles élémentaires de Hesse électorale dans la deuxième moitié du siècle, donc pratiqué par plusieurs générations d'élèves ${ }^{(65)}$, comportait, dans son chapitre sur l'histoire du territoire, un paragraphe sur les «guerres de libération». Le fil conducteur de ces quelques lignes était à la fois la toute-puissance et la protection paternelle de Dieu dans les turbulences historiques de la monarchie. En effet, si les territoires étaient tombés entre les mains des Français et que le souverain avait dû fuir le pays, c'était parce que Dieu avait voulu punir les sujets. Et si Napoléon avait pu être chassé du trône, c'était toujours grâce à Dieu. Car c'était lui qui avait apporté le secours nécessaire pour y parvenir. C'était donc lui aussi, qui avait permis au souverain de revenir dans ses terres ${ }^{(66)}$. Permettant de passer sous silence le côté compromettant de la période française ainsi que la défaite de 1813, la transcendance des événements révélait à la fois le rôle fondamentalement politique de la religion à l'école élémentaire ainsi que sa fonction dans la construction (et l'oubli) de l'histoire ${ }^{(67)}$.

À partir de 1871, un deuxième cadre de référence s'imposa pour les manuels d'une partie de la Confédération germanique: l'Empire allemand. Prenant le relais de la " patrie extérieure » dans sa version diminuée, ce nouveau cadre imprégna la représentation des "guerres de libération » dans les manuels, sans pour autant la changer complètement. Dans les manuels bavarois par exemple, la Bavière figura désormais parmi les puissances qui avaient libéré l'Allemagne tandis que les liens que le royaume avait entretenus avec la France à l'heure napoléonienne furent passés sous silence. Ceci était d'autant plus simple qu'en 1813, la Bavière avait changé de camp politique à temps et figurait ainsi parmi les vainqueurs ${ }^{(68)}$. Les manuels prussiens quant à eux insérèrent les "guerres de libération " dans un schéma narratif téléologique et victorieux, et en firent ainsi l'un des mythes fondateurs de l'unification allemande avec la Prusse comme principal acteur ${ }^{(69)}$.

Tout comme les autres livres scolaires, les manuels autrichiens racontaient une histoire victorieuse. Tout comme eux, ce récit fut intégré dans une histoire dynastique longue: en l'occurrence celle des Habsbourg. Ainsi, les guerres furent insérées dans une trame historique différente. De même, elles suivirent une autre chronologie. Alors qu'en Prusse et dans les autres États de la Confédération germanique la bataille de Leipzig fut considérée comme l'événement principal et décisif des " guerres de libération », c'est à la bataille victorieuse d'Aspern que les manuels autrichiens renvoyèrent les élèves. En revanche, contrairement aux autres livres, les manuels autrichiens présentaient les « guerres de libération » comme la libération de l'Europe monarchique

65 Sylvia Kesper-Biermann, Staat und Schule in Kurhessen 1813-1866, Göttingen, Vandenhoeck \& Ruprecht, 2001, p. 117.

Der Kurhessische Kinderfreund, ein Lesebuch vorzugsweise für die Mittelclassen der Volksschule, Cassel, 1846, p. 282.

67 A. Green, Fatherlands (note 5), p. 204.

68 A. Meissner, «Waffenbrüderschaft» (note 36), p. 856-863.

69 A. Meissner, Nationalisierung (note 2), p. 88-96. 
par l'Autriche, donc dans un cadre européen. Quant au terme de "patrie ", sa sémantique resta floue afin de permettre, dans le contexte de la monarchie multi-ethnique des Habsbourg, des identifications multiples ${ }^{(70)}$.

Par ailleurs, parallèlement à leur insertion dans les manuels d'histoire à proprement parler, les « guerres de libération » apparurent dans les supports scolaires utilisés dans d'autres matières que l'histoire. Dans les recueils de chant par exemple nous trouvons Was ist des Deutschen Vaterland? d'Ernst Moritz Arndt à côté de morceaux destinés à la patrie "intérieure ${ }^{(71)}$. Intégrés dans les programmes de littérature dans les écoles supérieures depuis les années 1830 , les poèmes ainsi que les pièces de théâtre des "guerres de libération » firent leur chemin dans les anthologies, comme les pièces de Theodor Körner ${ }^{(72)}$, et par là dans les salles de classe, bibliothèques scolaires et même dans les foyers familiaux des élèves de par le fait que les anthologies furent une récompense fréquente pour les meilleurs élèves ${ }^{(73)}$. Par conséquent, les productions écrites des "guerres de libération " rentrèrent, elles aussi, dans un répertoire standardisé, autorisé et diffusé au-delà du cadre scolaire ${ }^{(74)}$.

\section{Conclusion}

L'analyse des fêtes scolaires, de l'enseignement ainsi que des outils pédagogiques a révélé l'entrée lente et progressive des conflits dans les écoles des différents États allemands avec une apparition « anticipée » en Prusse dans les années 1820. En même temps, elle a dévoilé la pluralité ainsi que le caractère évolutif des enjeux culturels des guerres dans les établissements scolaires.

Si les « guerres de libération » devinrent progressivement des enjeux de la culture scolaire, c'est d'abord pour des raisons d'ordre politique. En fait, pendant toute la période étudiée, l'éducation devait conduire les élèves à une identification avec leur " patrie » ainsi qu'avec leur souverain ${ }^{(75)}$ et faire écran à tout ce qui pouvait, aux yeux de ceux qui détenaient le pouvoir, ébranler l'ordre établi; elle devait en d'autres termes contribuer à la stabilisation de l'ordre social et assurer la pérennité de la dynastie régnante. Le mot «patrie » pouvait alors avoir deux significations, étroite ou large ${ }^{(76)}$. Instrument de la mission politique de l'école, l'histoire pouvait se transmettre ailleurs que

70 Ibid., p. 288-294.

71 S. Kesper-Biermann, Staat (note 65), p. 122-123.

72 Georg JäGER, « Lehrplan und Fächerkanon der höheren Schulen », in: Handbuch der deutschen Bildungsgeschichte, t 3: 1800-1870. Von der Neuordnung Deutschlands bis zur Gründung des Deutschen Reiches, Munich, Beck, 1987, p. 191-221, ici p. 196.

73 Cf. les rapports suivants: Jahresbericht des Königlichen Gymnasiums zu Eisleben, von Ostern 1892 bis Ostern 1893, p. 46. URL: http://digital.ub.uni-duesseldorf.de/ulbdsp/periodical/pageview/ 4213341?query=theodor\%20k\%C3\%B6rner (31.10.2013); Jahresbericht der Oberrealschule zu Crefeld für das Schuljahr 1913, URL: http://digital.ub.uni-duesseldorf.de/ulbdsp/periodical/pageview/54662 09 ?query=schulfeiern\%20befreiungskriege (22.11.2013).

74 Ces recueils standardisés n'étaient pas une spécificité allemande. Voir l'exemple de la Grande-Bretagne: S. LeTHBRIDGe, « Wars» (note 11).

75 S. Kesper-Biermann, Staat (note 65), p. 108 sq., 122 sq.; Carl Kirsch, Das deutsche Volksschulrecht, t. 1, Leipzig, Mayer, 1854, p. 285.

76 Ibid., p. 282. Voir également S. Kesper-Biermann, Staat (note 65), p. 122. 
dans des cours d'histoire à proprement parler: à travers l'enseignement du chant, de l'allemand, de la religion ou encore dans l'étude de l'Antiquité ${ }^{(77)}$. C'est d'ailleurs dans ces autres matières que la " patrie extérieure " prenait souvent des formes concrètes pour les enfants. À travers les œuvres littéraires et musicales, ils se familiarisaient avec des représentations de ce qui était alors considéré comme étant authentiquement « allemand ${ }^{(78)}$.

Ensuite, les guerres de 1813 s'imposèrent pour des raisons pédagogiques et disciplinaires. Au-delà des années 1820, le champ de l'histoire cessa de se limiter au passé lointain et engloba le passé proche, divisant le temps révolu en trois époques chronologiques distinctes: l'Antiquité, le Moyen Âge et l'Histoire moderne (Neuere Geschichte), la dernière débutant soit avec la découverte des Amériques ${ }^{(79)}$, soit au moment de la réforme luthérienne. De surcroît, les rôles des différentes époques évoluèrent, eux aussi, l'Antiquité cédant à l'Histoire moderne la place qu'elle tenait jusque-là dans l'orientation des jeunes gens. C'est donc dans les archives de cette dernière que la jeunesse allemande trouverait ses repères désormais, et il se trouvait précisément que les guerres de 1813 avaient légué un énorme bagage de références imprimées aux générations futures. Toutefois, la " fin de l'histoire " resta à géométrie variable en fonction du contexte politique, aussi bien situable en amont de la Révolution française qu'au lendemain des « guerres de libération ${ }^{(80)}$.

\section{Zusammenfassung}

Der Beitrag beschäftigt sich mit der Geschichte der „Befreiungskriege“ in der longue durée und lenkt den Blick auf ein Vermächtnis, das bislang kaum beachtet wurde. Gemeint ist die Bedeutung, die die Kriege in der Enkulturation der Schüler in Deutschland im 19. Jahrhundert besaßen. Drei Felder werden dabei näher betrachtet: Schulfeiern, Unterricht und Lehrmaterialen wie Schulbücher, Liedersammlungen oder Anthologien. Die Analyse der drei Bereiche in langfristiger Perspektive veranschaulicht erstens, dass die Kriege in Preußen bereits in den 1820er Jahren Eingang in die Schulen fanden, ansonsten aber nur langsam und allmählich in den schulischen Alltag integriert wurden, zweitens, dass trotz nicht zu leugnender Annäherungen weiterhin unterschiedliche Deutungsmuster über die Kriege koexistierten, und drittens, dass die Deutung und Bedeutung der „Befreiungskriege“ in der Schule dem Wandel und der Instabilität der Rahmenbedingungen entsprechend vielfältig und dynamisch waren.

77 S. Kesper-Biermann, Staat (note 65), p. 240-241.

78 J. Loeber, Gymnasiastenzeit (note 12), p. 12.

79 Dans le manuel suivant par exemple: Wilhelm Püтz, Grundriss der Geographie und Geschichte der alten, mittlern und neuern Zeit für die oberre Klassen höherer Lehranstalten, t. 3: Die neuere Zeit, Coblence, Baedeker, 1872. 